\title{
Eicosapentaenoic acid shows anti- inflammatory effect via GPR120 in 3T3-L1 adipocytes and attenuates adipose tissue inflammation in diet-induced obese mice
}

\author{
Hodaka Yamada ${ }^{*}$, Tomio Umemoto ${ }^{1}$, Masafumi Kakei ${ }^{1}$, Shin-ichi Momomura ${ }^{1}$, Masanobu Kawakami²,
} San-e Ishikawa ${ }^{3}$ and Kazuo Hara ${ }^{1}$

\begin{abstract}
Background: Saturated fatty acids have been shown to cause insulin resistance and low-grade chronic inflammation, whereas unsaturated fatty acids suppress inflammation via G-protein coupled receptor 120 (GPR120) in macrophages. However, the anti-inflammatory effects of unsaturated fatty acids in adipocytes have yet to be elucidated. Hence, the aims of the present study were to evaluate the anti-inflammatory effects of eicosapentaenoic acid (EPA) via GPR120 in adipocytes.

Methods: We used $250 \mu \mathrm{M}$ palmitate as a representative saturated fatty acid. 3T3-L1 adipocytes were used for in vitro studies. We further evaluated the effect of EPA supplementation in a high-fat/high-sucrose (HFHS) diet-induced adipose tissue inflammatory mouse model.

Results: EPA attenuated palmitate-induced increases in inflammatory gene expression and NF-kB phosphorylation in 3T3-L1 adipocytes. Silencing of GPR120 abolished the anti-inflammatory effects of EPA. In GPR120 downstream signal analysis, EPA was found to decrease palmitate-induced increases in TAK1/TAB1 complex expression. EPA supplementation suppressed HFHS-induced crown-like structure formation in epididymal adipose tissue and altered macrophage phenotypes from $\mathrm{M} 1$ to $\mathrm{M} 2$ in the stromal vascular fraction. Moreover, the EPA-containing diet attenuated increases in adipose p-JNK and phospho-p65 NF-KB levels.

Conclusions: In conclusion, the findings of the present study demonstrate that EPA suppresses palmitate-induced inflammation via GPR120 by inhibiting the TAK1/TAB1 interaction in adipocytes. EPA supplementation reduced HFHS diet-induced inflammatory changes in mouse adipose tissues. These results demonstrate adipose GPR120 as a potential therapeutic target for decreasing inflammation.
\end{abstract}

Keywords: Palmitate, Adipocytes, G-protein coupled receptor 120, Eicosapentaenoic acid, Macrophage phenotype

\section{Background}

Obesity is known to contribute to the pathogenesis of metabolic syndrome and established risk factor for cardiovascular disease [1]. Obesity causes insulin resistance due to chronic low-grade inflammation [2, 3]. In obese states, plasma free fatty acids (FFAs) levels, particularly saturated

\footnotetext{
* Correspondence: hyamada0510@jichi.ac.jp

${ }^{1}$ First Department of Comprehensive Medicine, Jichi Medical University Saitama Medical Center, 1-847 Amanumacho, Omiya, Saitama 330-8503, Japan

Full list of author information is available at the end of the article
}

free fatty acids (SFAs) such as palmitate, are elevated and contribute to lipotoxicity in various organs [4]. On the other hand, polyunsaturated free fatty acids (PUFAs) such as eicosapentaenoic acid (EPA), an omega-3 ( $\omega-3)$ fatty acid, have been shown to have anti-inflammatory effects. Indeed, the long-term use of EPA supplementation in hypercholesterolemic Japanese patients reduced the risk of major coronary events [5]. Accordingly, FFAs are considered to have dual activity, namely inflammatory and anti-inflammatory effects. Recently, Oh and Olefsky et al. identified G-protein coupled receptor 120 (GPR120, Free 
Fatty Acid Receptor 4: FFAR4) as a receptor for $\omega-3$ fatty acids and demonstrated improved obesity-induced insulin resistance via GPR120 signaling in macrophages [6]. Oh et al. demonstrated that docosanexaenoic acid (DHA), a $\omega-3$ fatty acid, inhibits downstream nuclear factor- $\mathrm{B}$ (NF- $\kappa \mathrm{B})$ signaling via tumor necrosis factor- $\alpha$ (TNF- $\alpha$ ) receptor and toll-like receptor 4 (TLR4) activation in response to lipopolysaccharide (LPS) in macrophages [6, 7]. GPR120 is known to be expressed in adipose and intestinal tissues, in addition to macrophages $[6,8]$. Intestinal GPR120 activation increases secretion of glucagon-like peptide-1 and has been shown to have an anti-diabetic effect [8]. However, the mechanisms underlying anti-inflammatory effects of EPA treatment in adipocytes have yet to be elucidated. In the present study, we hypothesized that EPA directly affects adipocytes inflammation via GPR120 and ameliorates diet-induced obesity. The aims of the present study were to determine the anti-inflammatory effects of EPA via GPR120 in cultured adipocytes, and to clarify the effects of EPA supplementation on high-fat/high-sucrose (HFHS) diet-induced inflammatory changes in adipose tissue.

\section{Methods}

Reagents

Palmitate (Sigma-Aldrich) was used after conjugation with fatty acid-free albumin (Sigma-Aldrich). EPA sodium salt (EPA-Na) was purchased from Nu-Chek Prep, Inc. for cell treatment. Culture medium containing EPA was prepared according to previously described methods [9]. Highly purified EPA for mice was kindly provided by Mochida Pharmaceutical Company, Ltd.

\section{Cell culture, treatment, and harvesting}

3T3-L1 murine pre-adipocytes were purchased from American Type Tissue Culture Collection and differentiated according to a previously described standard protocol. [10] Fully differentiated 3T3-L1 adipocytes were exposed to $250 \mu \mathrm{M}$ palmitate for $30 \mathrm{~min}, 60 \mathrm{~min}$, or 24 $\mathrm{h}$ with or without a 6 -h pretreatment with $50 \mu \mathrm{M}$ EPA$\mathrm{Na}$. Cells were harvested by RIPA buffer with phosphatase and protease inhibitors (nacalai tesque) for protein extraction.

\section{Animal studies}

Male C57BL/6J mice (CLEA Japan, Inc.) were housed in accordance with our institutional guidelines and the Japanese Physiological Society's guidelines for animal care in an air-conditioned room with a 12-h light/dark cycle, with food and water available ad libitum. Mice were divided into three groups and fed a standard chow diet (MF diet, ORIENTAL YEAS CO.,LTD), high fat/ high sucrose (HFHS) diet (30\% fat, $20 \%$ sucrose) or HFHS diet supplemented with EPA (5\% wt/wt) for 24 weeks from 4 weeks of age. At the end of the study, mice were anesthetized by the intraperitoneal injection of pentobarbital $(100 \mathrm{mg} / \mathrm{kg})$ and then sacrificed. All experimental protocols for animal studies were approved by the institutional committee on animal care at Jichi Medical University.

\section{Plasma analysis}

Plasma insulin and adiponectin levels were measured by mouse insulin ELISA kits (Morinaga) and by adiponectin Bio-Plex Pro (BIO RAD), respectively. We performed blood glucose measurements at 12 and 18 weeks (Terumo Medisafe Mini Glucometer). Other plasma measurements were performed at Nagahama Life Science Laboratory (Shiga, Japan). The homeostasis model for insulin resistance (HOMA-IR) was calculated using the following formula: HOMA-IR = fasting plasma glucose $(\mathrm{mg} / \mathrm{dL}) \times$ fasting plasma insulin/405

\section{Stromal vascular fraction isolation}

The stromal vascular (SV) fraction was isolated from epididymal adipose tissue according to previously described methods with some modifications $[11,12]$. In brief, after sacrifice, epididymal adipose tissues were resected, minced $(<2 \mathrm{~mm})$, and digested with collagenase (Sigma-Aldrich) in Krebs-Henseleit-HEPES buffer, $\mathrm{pH} 7.4$, containing 20 $\mathrm{mg} / \mathrm{mL}$ of BSA and $2.8 \mathrm{mM}$ glucose at $37{ }^{\circ} \mathrm{C}$ using a shaker for $45 \mathrm{~min}$. Then, samples were passed through a $40-\mu \mathrm{m}$ mesh and centrifuged (1,000 g for $8 \mathrm{~min}$ ). Pellets and floating cells were collected as the SV fraction and primary adipocytes, respectively. Isolated cells were then used for RNA extraction.

\section{Transfection and Real-time quantitative reverse- transcription polymerase chain reaction}

RNA was isolated from 3T3-L1 adipocytes and primary adipocytes with Direct-zol ${ }^{\mathrm{TM}}$ RNA MiniPrep (Zymo Research). Complementary DNA was made with ReverTra Ace qPCR RT Master Mix (TOYOBO). Reverse transcription polymerase chain reaction (RT-PCR) was performed using TaqMan ${ }^{\oplus}$ gene expression assays (Applied Biosystems). Primer-probe sets are shown in Additional file 1: Table S1. All mRNA levels were normalized by GAPDH, as an internal control gene. Relative gene expression levels were calculated using the $\triangle \Delta C T$ method as previously described [13]. Gene silencing of GPR120 was performed using mouse GPR120 siRNA (Santa cruz) according to the manufacturer's protocol. The siRNA efficiency was determined using western blotting.

\section{Western blot analysis and co-immunoprecipitation}

Total cell lysates were obtained as previously described [10] and nuclear protein fractions were extracted using Universal Magnetic Co-IP kits (Active Motif) according to the manufacturer's protocol. Protein samples were resuspended 
in SDS sample buffer and boiled at $100{ }^{\circ} \mathrm{C}$ for $3 \mathrm{~min}$. The same volume of each sample was applied and separated by SDS-PAGE and electrophoretically transferred to polyvinylidene difluoride membranes. Membranes were immunoblotted using the following primary antibodies: tumor growth factor $\beta$ (TGF- $\beta$ ) activated kinase 1 (TAK1), TGF- $\beta$ activated kinase 1 binding protein 1 (TAB1) (Santa Cruz Biotechnology, SC-6052, lot: C1114), phospho-interferon regulatory factor 3 (IRF3) (Cell signaling, \#4947, lot: 7), total c-Jun NH2-terminal kinase (JNK) (Cell signaling, \#9252, lot: 15), phospho-JNK (Cell signaling, \#9251, lot: 21), NF-kB-p65 (Cell signaling, \#8242, lot: 4), and phosphoNF-кB-p65 (Cell signaling, \#3031, lot: 8), TNF receptorassociated factor 6 (TRAF6) (Invitrogen, 38-0900, lot: 1308635A), GPR120 (Abcam, ab97272, lot: GP26308-24). Co-immunoprecipitation to evaluate the interaction between TAK1 and TAB1 was performed using Universal Magnetic Co-IP kits (Active Motif) according to the manufacturer's protocol. Collected lysates and immune complexes were analyzed by western blotting. All signals were analyzed by C-Digit (LI-COR). GAPDH antibodies (Wako) were used as an internal control.

\section{Histological analyses}

Macrophage infiltration into epididymal adipose and hepatic tissues was evaluated using MAC-2 immunostaining. MAC-2 antibody staining and hematoxylin and eosin (HE) staining were performed at Biopathology Institute Co,. Ltd. (Oita, Japan). The number of crown-like structures (CLSs) was counted in five independent fields and recorded as the mean number of CLDs per low-power field (Olympus BX$51, \times 100)$. Adipocyte diameter was measured as the mean diameter of 100 adipocytes in each slide.

\section{Statistical analysis}

All data are shown as means \pm SEM. Statistical significance was determined using the unpaired Student's $t$-test.
Spearman's correlation was used for linear regression analysis. Prism version 5 (GraphPad software Inc.) was used for statistical analyses. $P$-values of $<0.05$ were considered statistically significant.

\section{Results}

Palmitate exposure increased monocyte chemoattractant protein-1 (MCP-1) and tumor necrosis factor (TNF)- $\alpha$ gene expression in 3T3-L1 adipocytes (Fig. 1). EPA pretreatment suppressed the expression of palmitateinduced inflammatory gene. Silencing of GPR120 abolished the anti-inflammatory effect of EPA; however, control scrambled siRNA did not demonstrate a significant effect (see Additional file 1: Figure S1A for silencing efficacy evaluation). GPR120 expression was not observed in pre-adipocytes, and palmitate exposure and EPA treatment had no effect on GPR120 protein expression levels (Additional file 1: Figure S1B). Palmitate induced the expression of p-IRF3, TRAF6 p-JNK, and downstream signals of TLR4 in cytosol, and phosphoNF-kB p65 in the nuclear fraction, of 3T3-L1 adipocytes (Fig. 2a). EPA pretreatment reduced p-IRF3, with a trend toward reduced $\mathrm{p}-\mathrm{JNK}$ and phospho-NF- $\mathrm{kB}$ p65 levels. Moreover, silencing of GPR120 tended to abolish this effect of EPA (Fig. 2b-e). Following palmitate treatment, Present or absent EPA treatment, and silencing of GPR120 also did not affect the expression levels of TAB1 (Fig. 3a). Co-immunoprecipitation of TAK1 and TAB1 demonstrated attenuation of palmitate-induced TAK1/TAB1 complexes in response to EPA pretreatment in the cytosolic fraction (Fig. 3b). These data indicate GPR120 signaling plays an inhibitory role in palmitate-induced inflammatory signal transduction and inflammatory gene expression in adipocytes.

Next, we examined the effect of EPA in HFHS diet-fed mice, an animal model of obesity and insulin resistance. Basic plasma parameters (after $6 \mathrm{~h}$ fasting) are shown in

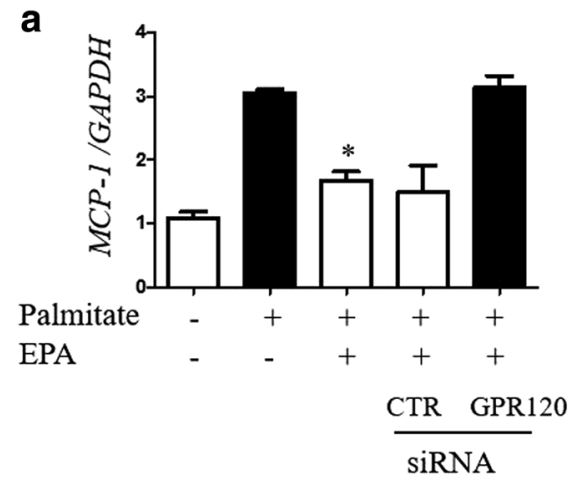

b

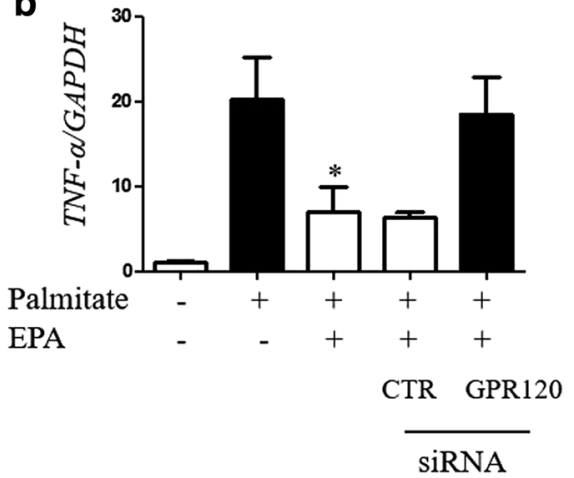

Fig. 1 mRNA expression of palmitate-induced MCP-1 (a) and TNF- $a(\mathbf{b})$ in 3T3-L1 adipocytes with or without GPR120 silencing. Before palmitate exposure, EPA pretreatment was performed. Data are presented as the mean \pm SEM of three independent experiments. ${ }^{*} P<0.05$ vs. palmitate exposure. CTR; scramble siRNA 


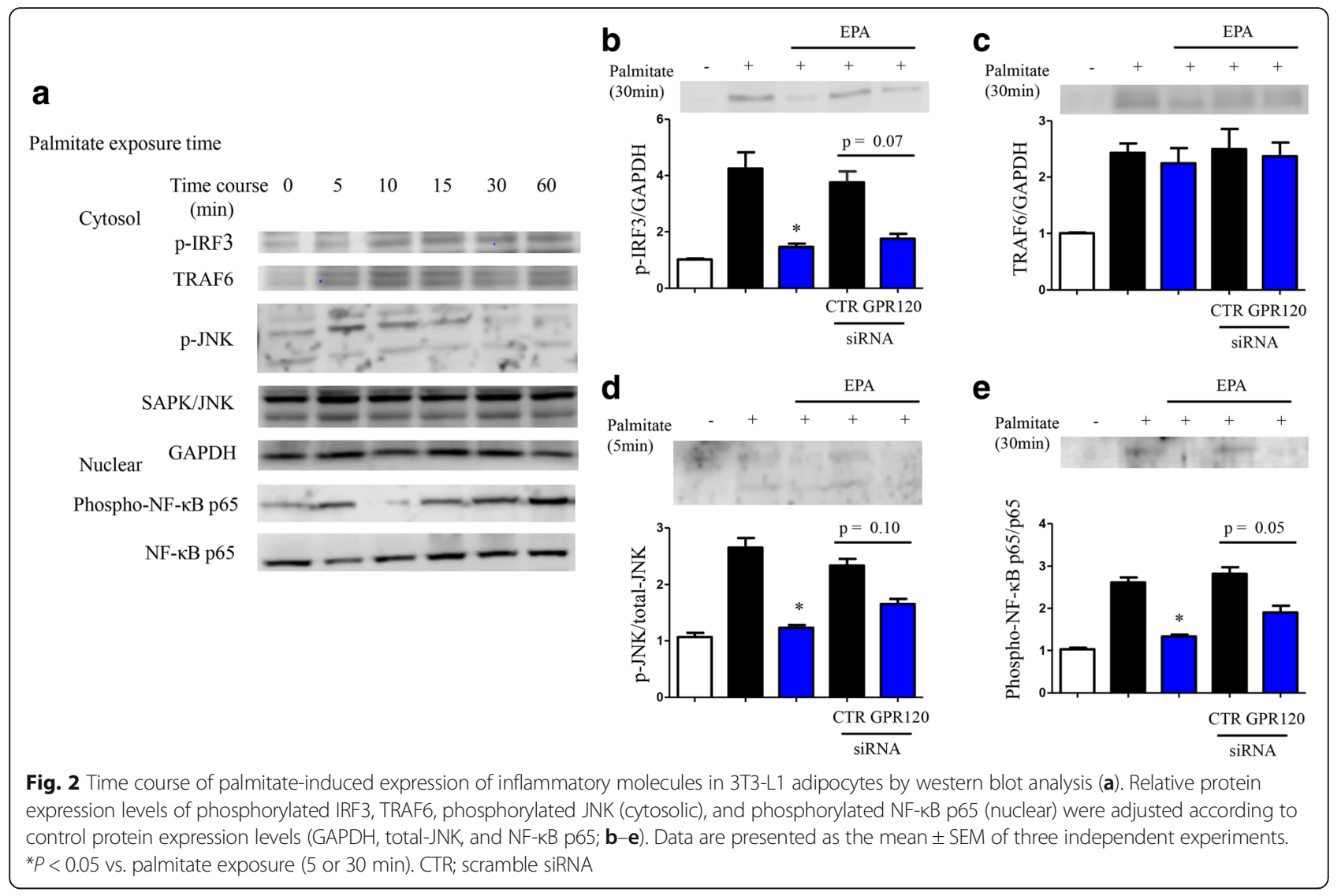

a

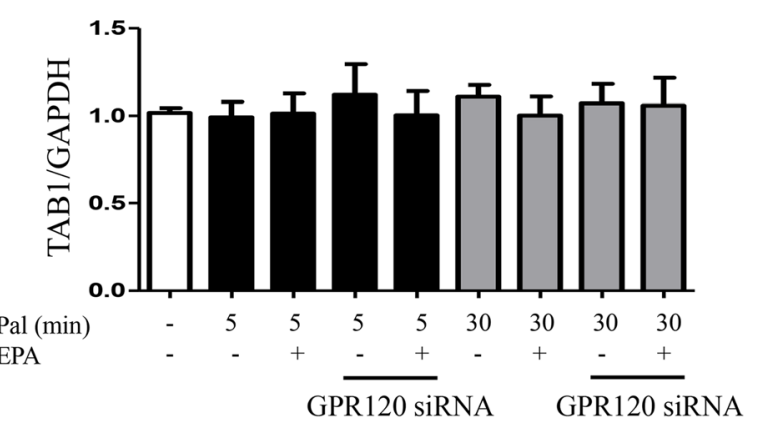

b
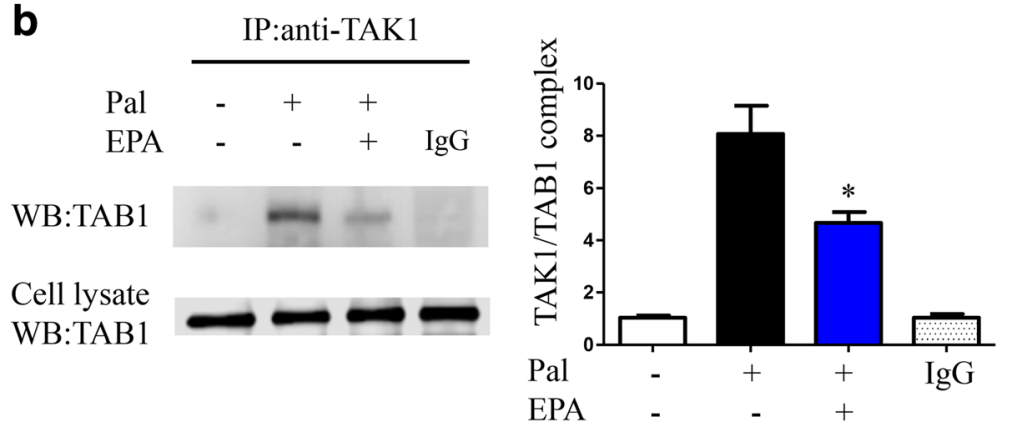

Fig. 3 TAB-1 protein expression levels after palmitate exposure (5 or $30 \mathrm{~min}$ ) with or without EPA pretreatment in transfected or non-transfected 3T3-L1 adipocytes (a). Co-immunoprecipitation analysis of TAK1 and TAB1 protein interaction (b). Data are presented as the mean \pm SEM of three independent experiments. ${ }^{*} P<0.05$ vs. palmitate exposure (30 min). IgG; control mouse lgG 
Table 1. EPA supplementation decreased plasma levels of total cholesterol, liver enzymes, and fasting plasma glucose levels. EPA significantly suppressed HFHS-diet induced body weight gain and reduced liver weight (\%g/ body), but did not affect epididymal mass (Fig. 4a-c). Fasting plasma glucose significantly reduced in the mice supplemented with HFHS containing EPA at 24 weeks (Fig. 4d). In addition, EPA supplementation improved HFHS-induced insulin resistance and increased plasma adiponectin concentration (Fig. 4e and f).

Histochemical analysis demonstrated EPA supplementation inhibited the formation of CLS in edididymal fat tissue (Fig. 5a-g). Overall, body weight (BW) and CLS number (/LPF) were found to be positively correlated $(\mathrm{r}$ $=0.80, P<0.001$, Fig. $5 \mathrm{~h}$ ), and CLS number was positively correlated with HOMA-IR $(r=0.72, P<0.001$, Fig. 5i). Moreover, EPA supplementation significantly decreased HFHS diet-induced mean adipocyte diameter expansion (Fig. 5j). Liver immunohistochemistry demonstrated the presence of hepatic steatosis in mice fed an HFHS diet containing EPA (Additional file 1: Figure S2).

We then determined inflammatory and antiinflammatory gene expression levels, and adipose tissue macrophages (ATMs) marker changes in epididymal adipose tissues. In the SV fraction, levels of CD11c, a marker of M1 macrophage, was decreased in response to EPA supplementation. In addition, gene expression levels of IL6 and TNF- $\alpha$ were also suppressed (Fig. $6 a-f)$. In edididymal adipose tissues, the EPA-containing diet inhibited HFHS diet-induced increases in MCP-1 expression, but had no effect on CD11c and CD206, M2 macrophage markers (Fig. 6g-l). Moreover, we examined representative beige-like markers of adipocytes, uncoupling protein 1 (UCP1) and PR domain containing (PRDM) 16. We found the increased levels of mRNA of UCP1 in EPA-fed epididymal adipose tissue (Additional file 1: Figure S3). Finally, we examined inflammatory signal transduction in mouse epididymal adipose tissues. EPA supplementation suppressed HFHS diet-mediated phosphorylation of IRF3 and JNK in the cytosolic fraction. Furthermore, NF- $\mathrm{KB}$ p65 phosphorylation in the nuclear fraction in mice fed the HFHS diet was inhibited by EPA supplementation (Fig. 7a-e). No difference in GPR120 protein levels was observed between the three diet groups (Fig. 7f).

\section{Discussion}

In the present study, we demonstrated that EPA suppressed palmitate-mediated expression of MCP-1 and TNF- $\alpha$ via GPR120 in cultured adipocytes. Moreover, EPA pretreatment reduced inflammatory signaling molecules mediated through suppression of TAK1/TAB1 complex formation. In our mouse model, EPA supplementation reduced HFHS diet-induced CLS formation, inflammatory signal transduction, and changed macrophage phenotype in adipose tissues. EPA demonstrated anti-inflammatory activity in macrophages was found to be GPR120-dependent [6]. Previous studies have reported that EPA inhibits palmitate-induced gene expression of MCP-1 or TNF- $\alpha$ in cultured macrophages $[9,14]$. Our present results indicate GPR120 signaling in response to EPA inhibits palmitate-induced inflammation in adipocytes. Palmitate is a major SFA and induces injury in endothelial cells [15], hepatocytes [16], adipocytes [14] and pancreatic $\beta$-cells [17]. SFAs are considered a ligand of TLR4, with SFA rich-diets shown to cause low-grade chronic inflammation resulting in insulin resistance [14, 17]. It is known that TLR4 signaling activates both myeloid differentiation primary response gene $(\mathrm{MyD})$ 88-depending pathway (TRAF6, JNK) and MyD88-independent pathway (IRF3) $[18,19]$. In this study, we found that EPA inhibited these downstream signals transduction of TLR4, of note, GPR 120 silencing tended to abolish these anti-inflammatory actions of EPA. Song et al. showed low dose palmitate $(10 \mu \mathrm{M})$ activated extracellular signal-regulated kinase (ERK)1/2, a mitogen-activated protein (MAP) kinase signaling, phosphorylation in GPR120 transfected HEK293T cells [20]. Oh et al. revealed that palmitate $(100 \mu \mathrm{M})$ also activated ERK phosphorylation in GPR120-knockdown RAW246 cells. On the other hand,

Table 1 Fasting plasma metabolic parameters

\begin{tabular}{lccc}
\hline & chow diet $(n=10)$ & $H F H S$ diet $(n=13)$ & HFHS diet + EPA $(n=8)$ \\
\hline TC $(\mathrm{mg} / \mathrm{dL})$ & $67 \pm 3.1$ & $181 \pm 14^{*}$ & $81 \pm 9.6^{\#}$ \\
TG $(\mathrm{mg} / \mathrm{dL})$ & $28 \pm 3.7$ & $13 \pm 1.6^{*}$ & $12 \pm 2.0$ \\
HDL-C (mg/dL) & $38 \pm 2.1$ & $63 \pm 3.9^{*}$ & $50 \pm 5.5$ \\
NEFA ( $\mu$ Eq/L) & $884 \pm 99$ & $630 \pm 47^{*}$ & $625 \pm 79$ \\
ALT (IU/L) & $9.4 \pm 1.9$ & $122 \pm 23^{*}$ & $32 \pm 11^{\#}$ \\
Plasma glucose $(\mathrm{mg} / \mathrm{dL})$ & $106 \pm 9$ & $126 \pm 4^{*}$ & $79 \pm 5^{\#}$ \\
Insulin (ng/mL) & $1.58 \pm 0.02$ & $7.06 \pm 0.41^{*}$ & $4.15 \pm 0.83^{\#}$ \\
HbAc (\%) & $4.58 \pm 0.05$ & $4.51 \pm 0.05$ & $4.52 \pm 0.05$ \\
\hline
\end{tabular}

Data are expressed as means \pm SEM. TC total cholesterol, TG triglyceride, HDL-C high-density lipoprotein cholesterol, NEFA nonesterified fatty acid, ALT alanine aminotransferase, HbA1c glycated hemoglobin, HFHS high-fat/high-sucrose, EPA eicosapentaenoic acid ${ }^{*} P<0.05$ vs. chow diet, ${ }^{\#} P<0.05$ vs. HFHS diet 

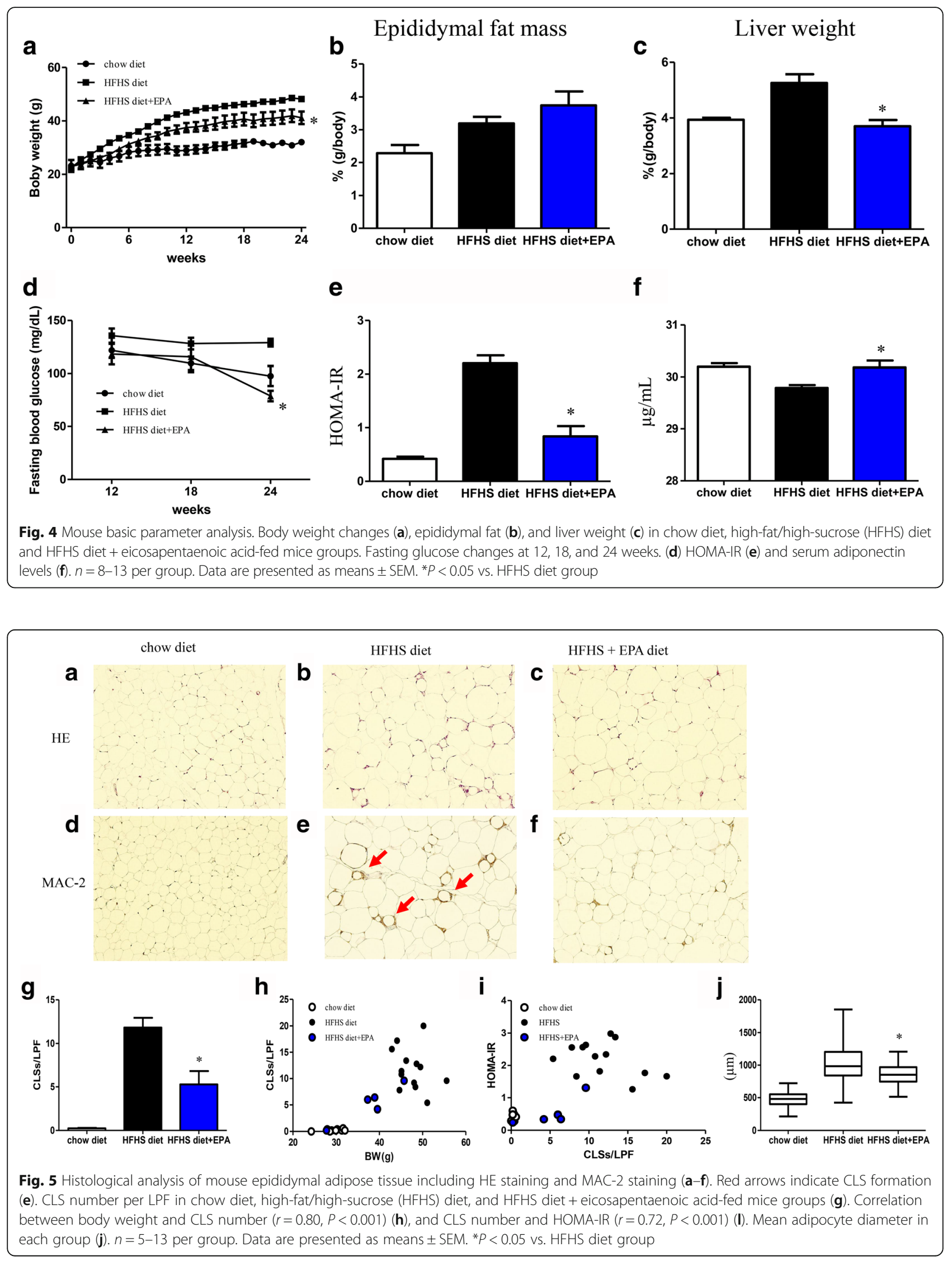

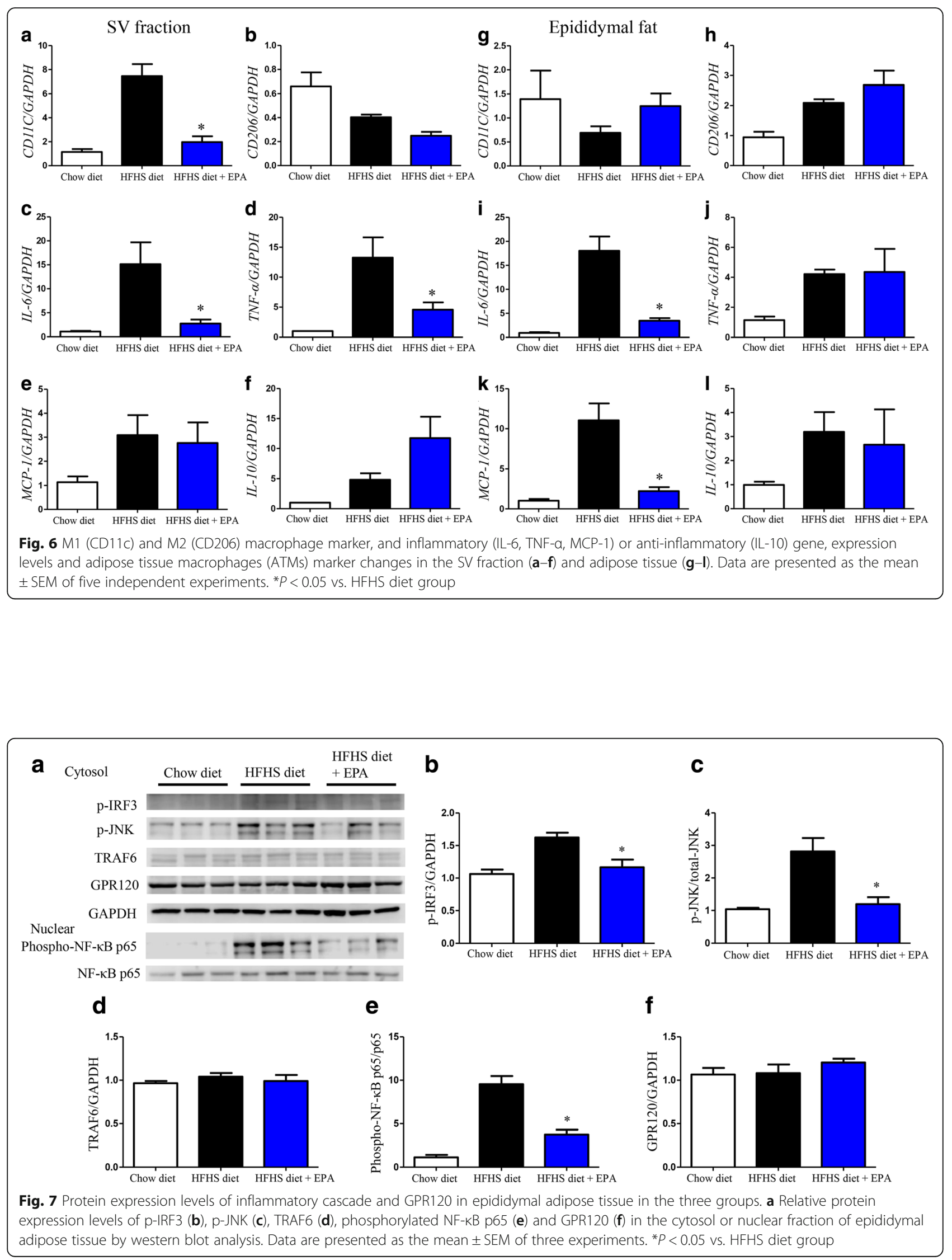
the action of docosahexaenoic acid (DHA) was abolished in GPR120-knockdown RAW246 cells [6]. We considered that $\omega-3$ fatty acids, such as DHA and EPA, show their action via GPR120, and saturated fatty acids including palmitate phosphorylates ERK independent of GPR120. In addition, our higher level of palmitate $(250 \mu \mathrm{M})$ might stimulate TNF and TLR4 signaling rather than GPR120 partial activation by palmitate. More detail studies are necessary to elucidate MAP kinase pathway signaling via GPR120.

TLR4 signal transduction also accelerates complex formation between TAK1 and TAB1, a key signal transducer of NF- $\kappa B$ in macrophages, and DHA inhibits TAK1/TAB1 complex formation [6]. In the present study, we demonstrate EPA has anti-inflammatory activity by inhibiting TAK1/TAB1 complex formation in 3T3-L1 adipocytes; however, protein expression of TAB1 did not change following palmitate exposure. These results indicate TAK1/TAB1 complex formation is more important than TAB1 protein expression levels. Recently, a GPR120-specific agonist was reported to reduce the interaction between TAK1 and TAB1 in intestinal epithelial cells [21]. Taken together, these results indicate GPR120-mediated anti-inflammatory pathways may represent a common system in GPR120-expressed tissues. In cultured human adipocytes, silencing of GPR120 has been shown to abolish the suppressive effect of TNF- $\alpha$ gene expression in response to DHA [22]. However, the results of the present study are not inconsistent with those of previous studies.

Numerous studies have reported EPA supplementation improves diet-induced fatty liver disease [23], obesity [24], and atherosclerosis [25] in mice. The results of the present study also demonstrate long-term EPA supplementation reduces BW gain, insulin resistance, and attenuates elevation of plasma alanine aminotransferase levels. CLS formation is known as a marker of the aggregation of M1 macrophages around dead or necrotic adipocytes in obese mouse models and humans [26].

As M1 macrophages are inflammatory bone marrow-derived macrophages, ATM polarity shifts from M2, a resident anti-inflammatory macrophage, to M1 macrophage phenotype in obesity. Fujisaka et al. reported that telmisartan (angiotensin II receptor blocker) or pioglitazone (Thiazolidinedione) reduces CLS formation and induces ATM polarity shifts (M1 to M2 macrophage) in diet-induced obese mice. Peroxisome proliferator-activated receptor $\gamma$ (PPAR $\gamma$ ) activation is thought to play an important role in ATM polarity shifting (M1 to M2 macrophage) as PPAR activation in response to telmisartan and pioglitazone has been shown to reduce adipocyte size [11, 27]. In obese humans, 12-week administration of omega-3acid ethyl esters has been shown to reduce CLS formation and MCP-1 gene expression in adipose tissue [28]. Indeed, EPA treatment increases the secretion of adiponectin by 3T3-L1 adipocytes, and plasma adiponectin levels in EPA-rich diet fed mice have been shown to be elevated $[29,30]$. Chambrier et al. reported EPA treatment $(50 \mu \mathrm{M}, 6 \mathrm{~h})$ increased mRNA levels of PPAR $\gamma$ in adipocytes isolated from humans [31]. We also observed a reduction in adipocyte size and increased plasma adiponectin concentrations in EPAcontaining HFHS diet-fed mice. Recent study of Pinel et al. revealed that EPA supplementation inhibited fat cell hypertrophy in visceral adipose tissue and improved the adiponectin expression. Our present study was not consistent with this previous examination [32]. Song et al. reported that GPR120 stimulation promote adipogenesis and triglyceride accumulation in cultured 3T3-L1 adipocytes [33]. These newly differentiated adipocytes might act as energy store and improve glucose tolerance [34]. In recent obesity classification, metabolically healthy obese (MHO) is proposed. MHO individuals present obesity, however, have no metabolic syndrome components with increased adiponectin and reduced visceral adiposity [35]. Klöting et al. showed that visceral adipocytes size and macrophage infiltration of $\mathrm{MHO}$ individuals are smaller and fewer than obese metabolic syndrome individuals [36]. We did not find the differences between HFHS diet and HFHS + EPA supplementation groups, however, taken together, newly differentiated adipocytes might attenuate adipocytes size, and improve lipid metabolism and insulin resistance [34].

In addition to the above-mentioned adipogenesis aspects, it has been found that white adipose tissue could change their phenotype by some stimulation [34]. UCP1 is a key protein to regulate energy expenditure of brown adipocytes and is also expressed in beige cells, which is induced from white adipose tissue [37]. It is known that beige cells are mitochondria-rich adipocytes and thermogenic activity, then could be inducible by cold, norepinephrine, irisin and fibroblast growth factor 21 stimulations [34]. Recently, EPA or fish oil induces UCP1 and changes the phenotype of white adipocytes to beige-like adipocytes [38, 39]. We found EPA supplementation increased UCP1 mRNA expression. EPA-induced beiging of adipocytes might contribute to the improvement of metabolic profile, however, whether these actions depends on GPR120 stimulation by EPA remains unclear. More detail study is needed to elucidate this point.

However, the potential effect of EPA on adiponectin protein or gene expression in vivo remains controversial. The above study reported the administration of omega-3-acid ethyl esters had no effect on adipocyte size or plasma adiponectin concentrations in a 
population of obese individuals [28]. These differences may be induced by EPA in vivo. In animal studies, dietary EPA doses are typically 5\% (wt/wt) or more. However, the administration of doses at these levels in daily dietary forms is clinically challenging. Accordingly, comparisons between animal and human studies should be interpreted with caution, especially among nutrient supplementation studies.

Otherwise, improved insulin resistance may be an effect of decreased MCP-1 gene expression in adipose tissue, thereby reducing the involvement of M1 macrophage in adipose tissue. HFHS diet-induced adipose inflammation may therefore be improved. We demonstrated EPA supplementation reduces expression of the M1 macrophage marker, CD11c, in the SV fraction. In fact, several in vitro and in vivo studies have reported that EPA-treatment inhibits palmitate-induced MCP-1 induction in cultured adipocytes [14] and HFHS dietinduced MCP-1 induction in adipose tissues [24]. A direct effect of EPA on adipocytes may contribute to antiinflammatory action via suppression of MCP-1 gene expression in adipose tissues. Oh et al. demonstrated that $\omega-3$ fatty acid supplementation has no effect in macrophage specific GPR120 knock-down mice [6]. However, whether the effect of ATM polarity shift depends on macrophage or adipose GPR120 has yet to be elucidated.

In the present study, EPA inhibited HFHS diet-induced inflammation. Sato et al. reported similar results, with 20 weeks of EPA supplementation found to suppress BW gain and MCP-1 gene expression in HFHS diet-fed mice, but to have no effect in high-fat (HF) diet-fed mice [24].

Sucrose is an independent risk factor for metabolic syndrome and non-alcoholic fatty liver disease (NAFLD) [40, 41]. We also observed that EPA supplementation inhibits HFHS-mediated steatosis and CLS formation in mouse liver (Additional file 1: Figure S2), and decreases the lipid fraction of LDL (Additional file 1: Figure S4 and Table S2). HFHS diet has been shown to induce more severe hepatic triglyceride accumulation and hepatic lipogenesis [24]. In addition, oral administration of EPA affects systemic metabolism. Further studies are required to elucidate the organ-specific agonistic effect of GPR120.

\section{Conclusions}

In the present study, we demonstrate EPA treatment inhibits TAK1/TAB1 complex formation and inflammatory responses via GPR120 in cultured adipocytes. In addition, EPA supplementation reduced HFHS dietinduced CLS formation and MCP-1 gene expression in mouse adipose tissues. These results indicate adipose GPR120 plays an important role in anti-inflammatory activity.

\section{Additional file}

\begin{abstract}
Additional file 1: Figure S1. Efficacy of siRNA for GPR120 (a) and GPR120 protein expression analysis in 3T3-L1 adipocytes (CTR, eicosapentaenoic acid 6h, palmitate $24 \mathrm{~h}$ exposure) and preadipocytes by western bot (b). Figure S2. Liver histological analysis by HE staining and MAC-2 staining $(\times 100)$. Red arrows indicated hepatic CLS formation. Figure S3. EPA supplementationinduced beige-like marker in epididymal adipose tissue. mRNA expression of uncoupling protein 1 (UCP1) and PR domain containing (PRDM) 16 was evaluated in each diet groups. Data are presented as the mean \pm SEM of five independent experiments. Figure S4. Cholesterol and triglyceride profile by HPLC. Lipid fraction of chow diet (a), high-fat/high-sucrose (HFHS) diet (b), and HFHS diet + eicosapentaenoic acid (c). The blue line represents triglyceride and pink line represents cholesterol contents. The LDL peak was found in HFHS diet plasma (b). VLDL, very low-density lipoprotein; LDL, low-density lipoprotein; HDL, high-density lipoprotein; FG, free glycerol. Method of this analysis is described in the following supplementary method. Table S1. Taq Man ${ }^{\circledR}$ Gene Expression ID information. Table S2. Analysis of plasma lipoproteins by HPLC. (PDF 497 kb)
\end{abstract}

\section{Abbreviations \\ ATMs: Adipose tissue macrophages; CLSs: Crown-like structures; \\ DHA: Docosanexaenoic acid; EPA: Eicosapentaenoic acid; FFAs: Free fatty acids; GPR120: G-protein coupled receptor 120; HFHS: High-fat/high-sucrose; HOMA-IR: Homeostasis model for insulin resistance; IRF3: Interferon regulatory factor 3; JNK: C-Jun N-terminal kinase; LPS: Lipopolysaccharide; MCP-1: Monocyte chemoattractant protein-1; NF-kB: Nuclear Factor-kB; PPARy: Peroxisome proliferator-activated receptor $\gamma$; PUFAs: polyunsaturated free fatty acids; SFAs: saturated free fatty acids; TAB1: TGF- $\beta$ activated kinase 1 binding protein 1 ; TAK1: TGF- $\beta$ activated kinase 1 ; TGF- $\beta$ : tumor growth factor $\beta$; TLR4: toll-like receptor 4; TNF-a: tumor necrosis factor-a; TRAF6: TNF receptor associated factor 6}

\section{Acknowledgments}

The authors thank Ms. Harue Fukaya, Taeko Ohtani, Chizuru Kobayashi, Tomoko Takahara, and Sachimi Jimbo at Jichi Medical University Saitama Medical Center for their technical assistance.

\section{Funding}

This work was supported by a Grant-in-Aid for Scientific Research (C) (5001-89) from the Japan Society for the Promotion of Science (JSPS) to T.U., and JMU Graduate Student Start-Up Grant for Young Investigators to H.Y. This study was subsidized by JKA through its promotion funds from KEIRIN RACE to Jichi Medical University Saitama Medical Center.

Availability of data and materials

All datasets are included within this article.

\section{Authors' contributions}

HY planned research design, collected the data, and wrote the manuscript. TU collected and analyzed data. MKakei, SM, MKawakami, SI, and KH analyzed data and edited the manuscript.

\section{Competing interests}

The authors declare that they have no competing interests

Consent for publication

All authors read and approved the final manuscript.

\section{Ethics approval}

This study was carried out in accordance with the Japanese Physiological Society's guidelines for animal care. All experimental protocols for animal studies were approved by the institutional committee on animal care at Jichi Medical University.

\section{Publisher's Note}

Springer Nature remains neutral with regard to jurisdictional claims in published maps and institutional affiliations. 


\section{Author details}

'First Department of Comprehensive Medicine, Jichi Medical University Saitama Medical Center, 1-847 Amanumacho, Omiya, Saitama 330-8503, Japan. ${ }^{2}$ Internal Medicine, Nerima Hikarigaoka Hospital, 2-11-1 Hikarigaoka, Nerima, Tokyo 179-0072, Japan. ${ }^{3}$ Division of Endocrinology and Metabolism, International University of Health and Welfare Hospital, 537-3 Iguchi, Nasushiobara, Tochigi 329-2763, Japan

\section{Received: 7 December 2016 Accepted: 1 May 2017} Published online: 08 May 2017

\section{References}

1. Matsuzawa Y. Establishment of a concept of visceral fat syndrome and discovery of adiponectin. Proc Jpn Acad Ser B Phys Biol Sci. 2010;86:131-41.

2. Boutens L, Stienstra R. Adipose tissue macrophages: going off track during obesity. Diabetologia. 2016:59:879-94

3. Gregor MF, Hotamisligil GS. Inflammatory mechanisms in obesity. Annu Rev Immunol. 2011:29:415-45.

4. Holzer RG, Park EJ, Li N, Tran H, Chen M, Choi C, Solinas G, Karin M. Saturated fatty acids induce c-Src clustering within membrane subdomains, leading to JNK activation. Cell. 2011;147:173-84.

5. Yokoyama M, Origasa H, Matsuzaki M, Matsuzawa $Y$, Saito $Y$, Ishikawa $Y$, Oikawa S, Sasaki J, Hishida H, Itakura H, et al. Effects of eicosapentaenoic acid on major coronary events in hypercholesterolaemic patients (JELIS): a randomised open-label, blinded endpoint analysis. Lancet. 2007;369:1090-8.

6. Oh DY, Talukdar S, Bae EJ, Imamura T, Morinaga H, Fan W, Li P, Lu WJ, Watkins SM, Olefsky JM. GPR120 is an omega-3 fatty acid receptor mediating potent anti-inflammatory and insulin-sensitizing effects. Cell. 2010;142:687-98.

7. Talukdar S, Olefsky JM, Osborn O. Targeting GPR120 and other fatty acidsensing GPCRs ameliorates insulin resistance and inflammatory diseases. Trends Pharmacol Sci. 2011;32:543-50.

8. Hirasawa A, Tsumaya K, Awaji T, Katsuma S, Adachi T, Yamada M, Sugimoto Y, Miyazaki S, Tsujimoto G. Free fatty acids regulate gut incretin glucagonlike peptide-1 secretion through GPR120. Nat Med. 2005:11:90-4.

9. Nakakuki M, Kawano H, Notsu T, Imada K. Eicosapentaenoic acid suppresses palmitate-induced cytokine production by modulating long-chain acyl-CoA synthetase 1 expression in human THP-1 macrophages. Atherosclerosis. 2013:227:289-96.

10. Umemoto T, Han CY, Mitra P, Averill MM, Tang C, Goodspeed L, Omer M, Subramanian S, Wang S, Den Hartigh $L$, et al. Apolipoprotein Al and highdensity lipoprotein have anti-inflammatory effects on adipocytes via cholesterol transporters: ATP-binding cassette A-1, ATP-binding cassette G-1, and scavenger receptor B-1. Circ Res. 2013;112:1345-54

11. Fujisaka S, Usui I, Bukhari A, Ikutani M, Oya T, Kanatani Y, Tsuneyama K, Nagai Y, Takatsu K, Urakaze M, et al. Regulatory mechanisms for adipose tissue M1 and M2 macrophages in diet-induced obese mice. Diabetes. 2009:58:2574-82

12. Nishimura S, Manabe I, Nagasaki M, Eto K, Yamashita H, Ohsugi M, Otsu M Hara K, Ueki K, Sugiura S, et al. CD8+ effector T cells contribute to macrophage recruitment and adipose tissue inflammation in obesity. Nat Med. 2009;15:914-20.

13. Jia L, Vianna CR, Fukuda M, Berglund ED, Liu C, Tao C, Sun K, Liu T, Harper MJ, Lee CE, et al. Hepatocyte Toll-like receptor 4 regulates obesity-induced inflammation and insulin resistance. Nat Commun. 2014;5:3878.

14. Yeop Han C, Kargi AY, Omer M, Chan CK, Wabitsch M, O'Brien KD, Wight TN, Chait A. Differential effect of saturated and unsaturated free fatty acids on the generation of monocyte adhesion and chemotactic factors by adipocytes: dissociation of adipocyte hypertrophy from inflammation. Diabetes. 2010;59:386-96.

15. Kim F, Pham M, Luttrell I, Bannerman DD, Tupper J, Thaler J, Hawn TR, Raines EW, Schwartz MW. Toll-like receptor-4 mediates vascular inflammation and insulin resistance in diet-induced obesity. Circ Res. 2007; 100:1589-96.

16. Egnatchik RA, Leamy AK, Noguchi Y, Shiota M, Young JD. Palmitate-induced activation of mitochondrial metabolism promotes oxidative stress and apoptosis in H4IIEC3 rat hepatocytes. Metabolism. 2014;63:283-95.

17. Eguchi K, Manabe I, Oishi-Tanaka Y, Ohsugi M, Kono N, Ogata F, Yagi N, Ohto U, Kimoto M, Miyake K, et al. Saturated fatty acid and TLR signaling link beta cell dysfunction and islet inflammation. Cell Metab. 2012;15:518-33.
18. Li X, Jiang S, Tapping RI. Toll-like receptor signaling in cell proliferation and survival. Cytokine. 2010:49:1-9.

19. Lu YC, Yeh WC, Ohashi PS. LPS/TLR4 signal transduction pathway. Cytokine. 2008;42:145-51.

20. Song T, Peng J, Ren J, Wei HK, Peng J. Cloning and characterization of spliced variants of the porcine $\mathrm{G}$ protein coupled receptor 120. Biomed Res Int. 2015;2015:813816

21. Anbazhagan AN, Priyamvada S, Gujral T, Bhattacharyya S, Alrefai WA, Dudeja PK, Borthakur A. A novel anti-inflammatory role of GPR120 in intestinal epithelial cells. Am J Physiol Cell Physiol. 2016;310:C612-621.

22. Rodriguez-Pacheco F, Gutierrez-Repiso C, Garcia-Serrano S, Alaminos-Castillo MA, Ho-Plagaro A, Valdes S, Garcia-Arnes J, Gonzalo M, Andrade RJ, MorenoRuiz FJ, et al:: The pro-/anti-inflammatory effects of different fatty acids on visceral adipocytes are partially mediated by GPR120. Eur J Nutr 2016.

23. Suzuki-Kemuriyama N, Matsuzaka T, Kuba M, Ohno H, Han SI, Takeuchi Y, Isaka M, Kobayashi K, Iwasaki H, Yatoh S, et al. Different Effects of Eicosapentaenoic and Docosahexaenoic Acids on Atherogenic High-Fat Diet-Induced Non-Alcoholic Fatty Liver Disease in Mice. PLoS One. 2016;11: e0157580.

24. Sato A, Kawano H, Notsu T, Ohta M, Nakakuki M, Mizuguchi K, Itoh M, Suganami T, Ogawa Y. Antiobesity effect of eicosapentaenoic acid in highfat/high-sucrose diet-induced obesity: importance of hepatic lipogenesis. Diabetes. 2010;59:2495-504.

25. Matsumoto M, Sata M, Fukuda D, Tanaka K, Soma M, Hirata Y, Nagai R. Orally administered eicosapentaenoic acid reduces and stabilizes atherosclerotic lesions in ApoE-deficient mice. Atherosclerosis. 2008;197:524-33.

26. Cinti S, Mitchell G, Barbatelli G, Murano I, Ceresi E, Faloia E, Wang S, Fortier M, Greenberg AS, Obin MS. Adipocyte death defines macrophage localization and function in adipose tissue of obese mice and humans. J Lipid Res. 2005:46:2347-55.

27. Fujisaka S, Usui I, Kanatani Y, Ikutani M, Takasaki I, Tsuneyama K, Tabuchi Y

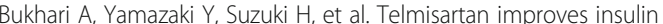
resistance and modulates adipose tissue macrophage polarization in highfat-fed mice. Endocrinology. 2011;152:1789-99.

28. Spencer M, Finlin BS, Unal R, Zhu B, Morris AJ, Shipp LR, Lee J, Walton RG, Adu $A$, Erfani $R$, et al. Omega-3 fatty acids reduce adipose tissue macrophages in human subjects with insulin resistance. Diabetes. 2013;62:1709-17.

29. Lefils-Lacourtablaise J, Socorro M, Geloen A, Daira P, Debard C, Loizon E, Guichardant M, Dominguez Z, Vidal H, Lagarde M, Bernoud-Hubac N. The eicosapentaenoic acid metabolite 15-deoxy-delta(12,14)-prostaglandin J3 increases adiponectin secretion by adipocytes partly via a PPARgammadependent mechanism. PLoS One. 2013;8:e63997.

30. Flachs P, Mohamed-Ali V, Horakova O, Rossmeisl M, Hosseinzadeh-Attar MJ, Hensler M, Ruzickova J, Kopecky J. Polyunsaturated fatty acids of marine origin induce adiponectin in mice fed a high-fat diet. Diabetologia. 2006;49:394-7.

31. Chambrier C, Bastard JP, Rieusset J, Chevillotte E, Bonnefont-Rousselot D, Therond P, Hainque B, Riou JP, Laville M, Vidal H. Eicosapentaenoic acid induces mRNA expression of peroxisome proliferator-activated receptor gamma. Obes Res. 2002;10:518-25.

32. Pinel A, Pitois E, Rigaudiere JP, Jouve C, De Saint-Vincent $S$, Laillet $B$ Montaurier C, Huertas A, Morio B, Capel F. EPA prevents fat mass expansion and metabolic disturbances in mice fed with a Western diet. J Lipid Res. 2016:57:1382-97.

33. Song T, Zhou Y, Peng J, Tao YX, Yang Y, Xu T, Peng J, Ren J, Xiang Q, Wei H. GPR120 promotes adipogenesis through intracellular calcium and extracellular signal-regulated kinase 1/2 signal pathway. Mol Cell Endocrinol. 2016;434:1-13.

34. Rosen ED, Spiegelman BM. What we talk about when we talk about fat. Cell. 2014;156:20-44

35. Denis GV, Obin MS. 'Metabolically healthy obesity': origins and implications. Mol Aspects Med. 2013:34:59-70.

36. Kloting N, Fasshauer M, Dietrich A, Kovacs P, Schon MR, Kern M, Stumvoll M, Bluher M. Insulin-sensitive obesity. Am J Physiol Endocrinol Metab. 2010;299: E506-515.

37. Wu J, Bostrom P, Sparks LM, Ye L, Choi JH, Giang AH, Khandekar M, Virtanen KA, Nuutila P, Schaart G, et al. Beige adipocytes are a distinct type of thermogenic fat cell in mouse and human. Cell. 2012;150:366-76.

38. Laiglesia LM, Lorente-Cebrian S, Prieto-Hontoria PL, Fernandez-Galilea M, Ribeiro SM, Sainz N, Martinez JA, Moreno-Aliaga MJ. Eicosapentaenoic acid promotes mitochondrial biogenesis and beige-like features in subcutaneous adipocytes from overweight subjects. J Nutr Biochem. 2016;37:76-82. 
39. Kim M, Goto T, Yu R, Uchida K, Tominaga M, Kano Y, Takahashi N, Kawada T. Fish oil intake induces UCP1 upregulation in brown and white adipose tissue via the sympathetic nervous system. Sci Rep. 2015;5:18013.

40. Malik VS, Popkin BM, Bray GA, Despres JP, Hu FB. Sugar-sweetened beverages, obesity, type 2 diabetes mellitus, and cardiovascular disease risk. Circulation. 2010;121:1356-64.

41. Bray GA, Popkin BM. Dietary sugar and body weight: have we reached a crisis in the epidemic of obesity and diabetes?: health be damned! Pour on the sugar. Diabetes Care. 2014;37:950-6.

Submit your next manuscript to BioMed Central and we will help you at every step:

- We accept pre-submission inquiries

- Our selector tool helps you to find the most relevant journal

- We provide round the clock customer support

- Convenient online submission

- Thorough peer review

- Inclusion in PubMed and all major indexing services

- Maximum visibility for your research

Submit your manuscript at www.biomedcentral.com/submit 